\title{
Bookmakers et parieurs entre rue et tripot
}

From Gambling Hell to Street Corner: Bookmakers and Their Punters

\section{Emmanuel Roudaut}

\section{OpenEdition}

\section{Journals}

Édition électronique

URL : http://journals.openedition.org/rfcb/1600

DOI : $10.4000 /$ rfcb. 1600

ISSN : 2429-4373

Éditeur

CRECIB - Centre de recherche et d'études en civilisation britannique

Édition imprimée

Date de publication : 1 septembre 2003

ISSN : 0248-9015

Référence électronique

Emmanuel Roudaut, "Bookmakers et parieurs entre rue et tripot », Revue Française de Civilisation Britannique [En ligne], XII-3 | 2003, mis en ligne le 01 septembre 2003, consulté le 20 avril 2019. URL : http://journals.openedition.org/rfcb/1600; DOI : 10.4000/rfcb.1600

Ce document a été généré automatiquement le 20 avril 2019

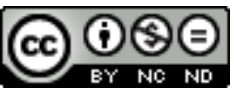

Revue française de civilisation britannique est mis à disposition selon les termes de la licence Creative Commons Attribution - Pas d'Utilisation Commerciale - Pas de Modification 4.0 International. 


\title{
Bookmakers et parieurs entre rue et tripot
}

\author{
From Gambling Hell to Street Corner: Bookmakers and Their Punters
}

\section{Emmanuel Roudaut}

1 Quelques années après le début du règne de Victoria, le monde des courses hippiques fut ébranlé par une série de scandales, à tel point que l'on se prit à redouter que le «sport des rois » ne suive le prize-fighting, c'est-à-dire le pugilat à mains nues, dans sa déchéance ${ }^{1}$ . Naguère prisé des élites, le prize fighting tomba rapidement en défaveur et disparut face à une répression accrue, tandis que se développaient les règles de la boxe moderne. Non seulement le Derby de 1844 fut terni par une fraude spectaculaire, mais le premier commissaire du Jockey Club, Lord George Bentinck, cadet d'une famille ducale, fut inquiété en vertu d'une loi sur les paris tombée en désuétude. Celle-ci fut opportunément suspendue tandis que deux commissions d'enquête parlementaire ${ }^{2}$, diligentées en 1844, devaient déboucher sur un remaniement complet de la législation sur les jeux et paris ( Gaming Act, 1845). La priorité, à l'époque, était de moraliser le turf, auquel la plupart des débats furent consacrés. Quant au monde du jeu, outre les champs de course, c'était sa face «nocturne » qui retenait l'attention, à savoir les gambling hells ou gambling dens, établissements clandestins où l'on se livrait à des jeux de hasard, souvent entre habitués fortunés. On s'intéressa fort peu à la persistance de combats d'animaux, qui donnaient pourtant lieu à de nombreux paris. En outre, la réforme de 1845 eut des conséquences inattendues, notamment le développement d'officines de paris urbains au comptant. Leur interdiction en 1853 n'empêcha pas la prolifération, sous des formes nouvelles désignées sous le terme générique de street betting, des paris urbains ${ }^{3}$ sur les courses hippiques dans les milieux populaires, si bien qu'à la fin de l'époque victorienne, c'était cette face «diurne» et populaire du jeu qui suscitait les principales inquiétudes, comme en témoignent les travaux d'une nouvelle enquête parlementaire en $1902^{4}$.

2 Le présent article tentera de retracer l'évolution des discours tenus sur le jeu, en l'abordant sous trois aspects. Après une rapide évocation des cercles de jeux, on s'intéressera aux craintes inspirées par les débordements associés aux hippodromes, ainsi qu'aux mesures prises pour éloigner ceux-ci des centres urbains. Sera ensuite abordé 
l'univers clandestin du street betting. Cette activité prohibée, loin de se cantonner à quelques tripots, semble avoir envahi l'espace public et fortement imprégné la culture populaire des villes victoriennes.

\section{Clubs et tripots}

3 Lord George Bentinck avait été poursuivi au prétexte d'une loi votée sous la reine Anne qui rendait illégale toute mise d'un montant supérieur à dix livres. Ce qui valait pour les courses hippiques s'appliquait aussi aux jeux de hasard (roulette, whist, baccara, etc.), comme le soulignait une question posée par la commission de 1844 à un autre membre éminent du Jockey Club, le capitaine Rous. Celui-ci s'empressa de faire la distinction entre deux types d'établissements, les clubs de gentlemen d'une part, et les 'common gambling houses' de l'autre. Il estimait déplacé de recourir à la loi pour la première catégorie, qu'il associait à des établissements prestigieux de St James's Street, tels White's, Brook's ou Boodle's ${ }^{5}$. S'il était de notoriété publique que des fortunes se jouaient et se perdaient quotidiennement dans ces clubs, leur premier mérite était d'être des établissements privés $^{6}$, de telle sorte que les transactions entre leurs membres ne tombaient pas sous le coup de la loi. En outre, il avait toute confiance dans la régularité des opérations conduites dans les clubs de St James tandis que selon lui, les autres établissements avaient systématiquement recours à des dés pipés ou à d'autres stratagèmes?

Ces établissements douteux, qu'à l'instar d'un membre de la commission et de la plupart de ses contemporains, il qualifiait de 'hells's, devaient quant à eux subir toutes les rigueurs de la loi $^{9}$. La police évaluait le nombre de ces tripots clairement identifiés à une quinzaine, tous situés dans le West End (paroisses de St George's, St James's, St Ann's et St Martin-in-the-Fields), mais ce chiffre n'incluait pas de nombreux établissements suspects, notamment des académies de billards, ainsi que des débits de tabac et de boisson (cigarshops and public houses $)^{10}$. D'autre part, les forces de l'ordre faisaient état de nombreuses entraves à leur action. Il était difficile de trouver des membres du voisinage disposés à faire une déclaration écrite sous serment, condition nécessaire à une perquisition, car il s'agissait souvent d'une pièce louée chez un particulier. Aux complexités de la procédure s'ajoutaient les manoeuvres d'intimidation, sans compter les guetteurs surveillant les déplacements des policiers ${ }^{11}$. Même quand une perquisition aboutissait, la configuration des lieux et l'interdiction de procéder à des fouilles corporelles laissaient aux joueurs le temps de dissimuler de l'argent ainsi que des objets compromettants (jeux de cartes, roulette, etc.), si bien qu'un magistrat déplorait la modicité des amendes infligées, fort peu dissuasives à son avis $^{12}$. Comme une perquisition ne pouvait être répétée sans renouveler toute la procédure, un établissement clandestin pouvait, dans la plupart des cas, reprendre impunément ses activités dès le lendemain de la visite des policiers. Ces doléances furent entendues et les pouvoirs de la police furent élargis par le Gaming Act de 1845 et le Gaming Houses Act de 1854.

5 Certains observateurs de l'époque soulignaient la diversité de ces tripots clandestins et distinguaient les 'respectable' gambling houses des 'low' gambling houses. Dans la première catégorie, où " un juif ou un individu mal vêtu n'aurait pas été admis », les sommes mises en jeu pouvaient être importantes, tandis qu'elles pouvaient se limiter à quelques pence dans la seconde ${ }^{13}$. En définitive, on peut donc parler de trois types d'établissements, qui correspondaient assez largement à une stratification sociale : les clubs de gentlemen, les common gaming houses « respectables» et celles qui étaient qualifiées d' "inférieures ». 
Seuls les clubs aristocratiques de St James échappaient aux poursuites judiciaires, la qualité de club privé étant généralement déniée aux autres établissements ${ }^{14}$. Les contemporains justifiaient cette disparité de traitement par la nécessité de protéger les pauvres et les classes moyennes de la déchéance. A cet égard, la déposition d'un policier reflétait une opinion courante :

I think that a penalty may be usefully enforced against certain classes, while others might be unmolested; because it is decidedly, I think, an evil for a spirit of gambling to prevail among the working and industrious community, while I should have no desire to interfere with that class of persons who, having ample funds and leisure, choose so to dispose of their property. I should think it a much graver evil for a working man to lose $5 \mathrm{l}$. in gambling than for the privileged classes to lose whatever they please ${ }^{15}$.

Derrière la mansuétude affichée à l'égard des «flambeurs » des grands clubs perçait toutefois une réprobation croissante, qui commença à se manifester par les propos tenus à l'encontre de Crockford's. Plus récent que ses voisins, ce club de St James's Street s'était ouvert en 1828. Le faste de l'établissement et la renommée de ses membres, qui incluaient le duc de Wellington, avaient assuré son succès dans la vie mondaine de la capitale ${ }^{16}$. Mais chacun connaissait les origines roturières de son propriétaire, William Crockford, fils de poissonnier dont une grande partie de la fortune provenait de ses activités de bookmaker. Grâce à ses moyens importants, la salle de jeux du nouveau club n'avait pas tardé pas à supplanter celles de tous ses rivaux, mais les nombreuses références à Crockford's au cours des travaux des commissions de 1844 étaient empreintes de suspicion. Si les membres du club étaient ménagés, sans doute en raison de leur origine sociale, de nombreux témoins, Rous en particulier ${ }^{17}$, mettaient en lumière, à travers le cas de Crockford's, la porosité de la frontière entre le monde des clubs aristocratiques et les gambling hells méprisés ${ }^{18}$. La sévérité de ces jugements traduisait le climat d'une époque. Des comportements admis sous la Régence ne l'étaient plus à l'époque du prince Albert et les historiens s'accordent à dire que la fréquentation des tables de jeu de St James's Street diminua fortement sous le règne de Victoria ${ }^{19}$.

\section{Courses hippiques ou saturnales?}

On ne peut dissocier les clubs et les tripots du monde des courses hippiques et des paris car on y retrouvait les mêmes personnes. Non seulement la plupart des membres de Brook's, White's et Crockford's assistaient aux «classiques » (courses de Newmarket et d'Ascot, Derby d'Epsom, Saint Léger à Doncaster, etc.), mais ils engageaient des paris plusieurs mois ou plusieurs semaines à l'avance (ante-post betting) dans leur club ${ }^{20}$ ou à Tattersall's, la plus importante salle londonienne de vente de chevaux. Quant aux tenanciers de maisons de jeux clandestines, aux dires des policiers, ils quittaient souvent la capitale pendant la saison des courses pour exercer leur activité sous des chapiteaux ou des baraques de foire sur les hippodromes ${ }^{21}$. La location de ces emplacements s'avérait indispensable au financement de la réunion, comme l'expliquait le commissaire des courses de Hampton ${ }^{22}$, reconnaissant qu'il traitait parfois avec des escrocs avérés ( thimbleriggers). Les loyers étant mis aux enchères, ceux des baraques de jeu étaient les plus élevés de tous, devançant même celui des chapiteaux où l'on servait des boissons. Seules les courses de Newmarket, entièrement financées par des membres du Jockey Club, pouvaient se passer de cette source de revenus. 
Les réunions hippiques, qui duraient plusieurs jours, étaient souvent associées à des fêtes locales traditionnelles (wakes weeks). Il régnait donc une atmosphère de fête foraine dans ces manifestations ouvertes à tous, où la course proprement dite ne constituait qu'une attraction parmi d'autres, comme le montre Derby Day, le célèbre tableau de William Powell Frith (1858). A la même époque, Hugh Shimmin nous a laissé une description sévère des courses d'Aintree ${ }^{23}$, qui étaient aux habitants de Liverpool ce que le Derby d'Epsom était aux Londoniens. Sa réprobation ne constituait pas une exception. De nombreux hommes d'église dénonçaient en chaire ce qu'ils percevaient comme des saturnales tandis que les ligues anti-alcooliques fustigeaient les incitations aux beuveries, dont les adeptes se faisaient ensuite dépouiller au jeu.

On déplorait surtout le relâchement des mœurs lors de ces festivités, notamment l'omniprésence de femmes excessivement fardées, euphémisme désignant les prostituées. Tout cela explique ce commentaire elliptique et consterné qu'entendirent les membres de la commission parlementaire de 1844 :

Question 1125: 'Are the Doncaster races attended by any great numbers of the lower

classes ?' - 'Immense masses.'

Q. 1126: 'Do you think more, or less of late years than formerly?' - 'More of late years.'

Q. 1127: 'Coming for the pleasure they derive from seeing the races?' - 'A little more than that, the mass of them. There is a great percentage who come for the other sensual gratifications provided for them at the races.'

Q. 1128: 'Eating and drinking, and so forth?' - 'And much worse.' ${ }^{24}$

L'affluence croissante observée par ce témoin était probablement liée au développement des chemins de fer, d'autant plus que des trains spéciaux furent régulièrement affrétés à l'occasion de rencontres hippiques dès la fin des années 1830. C'était le cas pour des courses importantes comme celles de Doncaster qui n'attiraient pas simplement la population locale. Le même témoin reconnaissait que depuis quelques années, sous la pression des autorités, les baraques de jeux avaient disparu de l'hippodrome, mais il ajoutait que pendant la semaine des courses, la ville était pleine de salles de jeux tenues par des thimble men venus de Londres ou de Manchester ${ }^{25}$. Il notait également l'attitude complaisante des commerçants locaux, qui redoutaient que la répression ne nuise aux retombées financières de cette semaine exceptionnelle.

11 Contraints de cesser de louer des emplacements aux cercles de jeux, les organisateurs se tournèrent vers des bookmakers professionnels afin qu'ils contribuent, en toute illégalité, au financement des réunions. On trouve des traces écrites de ces transactions remontant aux années 1860, ce qui n'exclut pas que des « loyers » aient été versés plus tôt ${ }^{26}$. A partir des années 1850 , les compagnies de télégraphe furent également mises à contribution. Les bookmakers semblent être devenus la principale cible des moralistes à mesure que l'évolution des courses permit la multiplication des paris et l'essor de leur profession au cours du dix-neuvième siècle.

Grâce au déclin de l'ancien système des matches, où une série d'éliminatoires débouchait sur un duel entre les deux meilleurs chevaux, il était désormais possible à un professionnel de prendre des paris sur tous les chevaux au départ (make a book) en minimisant ses risques. Les gains éventuels du parieur correspondaient à un multiple de sa mise, qui correspondait à la " cote » (odds ou price) du cheval ${ }^{27}$ Ces professionnels, dont l'origine sociale était bien inférieure à celle des aristocrates habitués du turf, étaient souvent tenus pour responsables de la recrudescence de scandales associés aux courses au début des années $1840^{28}$. S'ils opéraient parfois sous des tentes, la plupart des bookmakers 
œuvraient en plein air, aux abords de la piste et des tribunes. Il était impératif pour eux d'être aisément reconnaissables dans la foule, ce qui les conduisait à arborer des tenues assez voyantes, voire criardes. On en trouve une description dans cet encart publicitaire paru dans la presse sportive :

The gentlemen in high boots, sombrero hats, brown velvet coats and vests, with other tasty articles of dress...will attend, in the public ring, the above Meeting. This is on the authority of the gentleman whom racing men generally will recognise as Fred. Frazer and his genteel penciller... ${ }^{29}$

Un personnage de fiction, le bookmaker William Latch, arbore une tenue analogue pour se rendre au Derby ${ }^{30}$. Aujourd'hui encore, le personnage haut en couleur du 'on-course bookie' fait partie intégrante du spectacle des courses hippiques.

Un aspect moins pittoresque des hippodromes était la réputation de violence qui s'attachait à certains d'entre eux tout au long du dix-neuvième siècle. Un exemple bien connu est celui de Doncaster en 1829, où une tentative de mettre un terme aux activités illicites des thimblemen donna lieu à une bataille rangée entre ceux-ci et la police ${ }^{31}$. Il fallut le renfort de cavaliers pour que les forces de l'ordre aient le dessus. Il était plus fréquent de voir des altercations opposer des parieurs à des welshers, c'est-à-dire à des bookmakers véreux tentant de s'éclipser sans verser leur dû aux gagnants, notamment aux environs de Londres, où de nombreux hippodromes s'étaient créés au milieu du siècle, souvent à l'initiative de tenanciers de pubs et de bookmakers. Selon Vamplew, ces "saturnales de banlieue", où les courses étaient souvent truquées, "attiraient les éléments les plus indésirables de la capitale » et dégénéraient souvent en affrontements ${ }^{32}$. A titre d'exemple, l'auteur fait état d'une véritable bataille rangée entre des bookmakers véreux et leurs clients à Bromley. Des scènes analogues pouvaient se produire sur des hippodromes plus réputés. Ainsi James Greenwood relate le lynchage d'un welsher, survenu à Alexandra Park en $1868^{33}$. La foule s'en était prise aux policiers qui tentaient de s'interposer et la victime n'avait pas survécu à ses blessures. L'auteur dressait à cette occasion un tableau peu favorable d'une profession dominée selon lui par des escrocs ${ }^{34}$.

Ces scandales et ces violences avaient un effet dissuasif. Ainsi observait-on en 1844 que la bonne société, « lassée de voir les jeunes filles importunées par des blacklegs » ${ }^{35}$, que ce fût au bal ou sur les tribunes, avait déserté les courses de Doncaster. Les efforts du Jockey Club pour « moraliser » le turf, notamment sous la houlette de Lord George Bentinck à partir des années 1840 , n'eurent que peu d'effet en dehors de Newmarket. Ce n'est qu'à compter de 1870 qu'un nombre significatif de champs de courses acceptèrent de se plier aux directives publiées dans le Racing Calendar. La réglementation fut alors durcie: de 56 règles en 1876 , on passa à 184 en 1890 , si bien qu'à la fin du siècle, on s'accordait à reconnaître que le club avait renforcé son contrôle sur les courses les plus prestigieuses ${ }^{36}$.

Une autre des mesures prises était la sélection par l'argent. Dès le début du siècle, on édifia des tribunes dont l'accès était payant ${ }^{37}$. On fit de même pour une partie enclose du champ de courses. Vers la fin du siècle, on vit apparaître des champs de course entièrement clos, où chaque spectateur devait s'acquitter d'un droit d'accès. Le pionnier en la matière fut Sandown Park, ouvert en avril $1875^{38}$. Désormais, ce n'était plus une partie, mais l'ensemble du champ de courses qui était réservé à un public socialement «choisi». Quant aux hippodromes les plus mal famés des environs de Londres, le Racecourses Licensing Act de 1879 entraina leur disparition, car cette nouvelle loi subordonnait l'existence de tous les hippodromes situés dans un rayon de dix milles autour de Charing Cross à l'octroi par un juge de paix d'une licence renouvelable chaque 
année. Un déclin comparable à celui du prize ring, tant redouté au milieu du siècle, fut donc épargné aux courses hippiques, si bien qu'en 1909, un magazine sportif pouvait se féliciter de voir restaurée l'image de marque de ce sport:

Although it might be considered too optimistic to describe ruffianism as entirely a thing of the past, yet when the old stager thinks back twenty-five or thirty years to the palmy days of Croydon [etc.], it would really seem that racecourse hooliganism was almost non-existent. When Mr. Anderson's Racecourse Bill passed into law, and the 'park' meetings (Sandown, Kempton, etc.) came into being, with their wellpoliced enclosures, lawns and picturesque surroundings, the foul-mouthed blackguard, the three-card faker, the welsher and the tale-pitcher, found themselves, like Othello, with their occupation gone ${ }^{39}$.

\section{Les paris urbains entre ombre et lumière}

Les chevaux n'étaient pas les seuls animaux sur lesquels on engageait des paris. Ceux-ci se pratiquaient beaucoup à l'occasion de combats clandestins, notamment les combats de coqs, de chiens et ceux qui opposaient des chiens à des rats ou à des blaireaux. Leur prohibition, renforcée par le Cruelty to Animals Act de 1835, n'eut que des résultats mitigés. Le bull baiting et le bear baiting, naguère répandus, allaient bientôt disparaitre et il semble que la vogue des combats de coqs ait fortement décliné au milieu du siècle ${ }^{40}$. En revanche, celles des combats de chiens et du ratting, où l'on misait sur le nombre de rongeurs tués par le chien en un temps donné, ne faiblirent pas. On trouve chez Mayhew ${ }^{41}$ une description détaillée de ratting dans un pub londonien. Quelques années plus tard, Hugh Shimmin déclare qu'il ne se passe pas une semaine à Liverpool sans que plusieurs combats de chiens soient organisés par des tenanciers de débits de boissons. Pour celui qu'il relate, les cotes des animaux sont régulièrement annoncées comme on le ferait pour des pursang sur l'hippodrome ${ }^{42}$. Il ajoute qu'aux courses d'Aintree, une tente est réservée aux amateurs de la 'canine fancy', à côté de celles où se retrouvent les adeptes du pugilat ou du pédestrianisme ${ }^{43}$.

Loin d'avoir été éradiqués, les combats d'animaux ont perduré, à des degrés divers, jusqu'à nos jours. On ne saurait donc ignorer cet aspect de l'activité des parieurs et des bookmakers; toutefois l'époque victorienne fut principalement marquée par le développement des paris sur les courses hippiques, notamment dans les milieux populaires. Jusqu'à la régence, hormis quelques bookmakers comme William Crockford et John Gully, le monde du turf ne comptait qu'un cercle restreint de parieurs réguliers, issus pour la plupart de l'aristocratie, et les sommes misées pouvaient être considérables ${ }^{44}$ . Il n'en était pas de même pour les Derby Clubs et les sweepstakes, qui s'étaient répandus dans les pubs de nombreuses villes au cours des années 1840. A l'occasion de "classiques", on tirait au sort le nom du cheval sur lequel on misait. Cette pratique pouvait être assimilée à une loterie, dont la prohibition avait été renforcée en 1826 ; en outre, elle s'était démocratisée, car certains billets, au prix de quelques shillings, étaient désormais accessibles à de modestes employés ou artisans. C'est probablement pour cette raison que, sur intervention des autorités, les publicités pour les sweeps disparurent de la presse en $1848^{45}$. Au souci paternaliste de protéger les pauvres de la ruine s'ajoutait la crainte que les parieurs ne détroussent leur employeur pour régler leurs dettes.

Les poursuites engagées contre les sweepstakes favorisèrent l'essor de list houses dans des pubs londoniens, bientôt imités en province. Le bookmaker affichait les cotes offertes ( betting lists) sur les fenêtres de l'établissement, qui bénéficiait ainsi d'un surcroît 
d'activité, quand le tenancier ne faisait pas lui-même office de bookie. Comme la loi de 1845 avait ôté toute valeur légale aux dettes de jeu, les transactions se faisaient au comptant (ready-money betting) contre réception d'un ticket justificatif. Les gains étaient versés dès le lendemain de la course. Le succès fut indéniable: pour la seule ville de Londres, on parlait de plusieurs centaines de betting-shops, qui suscitaient les mêmes inquiétudes que les sweepstakes. Certains avançaient qu'elles constituaient une menace pour l'ordre social ${ }^{46}$, leur conférant ainsi l'image néfaste jusqu'alors dévolue aux seules gaming-houses. Une série de scandales, où de grands bookmakers disparaissaient sans payer les sommes dues, amenèrent le gouvernement à faire voter le Betting Act de 1853. Cette loi, qui allait rester en vigueur jusqu'en 1960, rendait les tenanciers de list houses, ancêtres des betting shops, passibles de peines pouvant aller jusqu'à l'emprisonnement.

Même avant 1853, des sporting public houses s'étaient vu refuser le renouvellement de leur licence, si bien qu'à l'instar des anciens gambling hells, des list houses s'étaient installés dans d'autres établissements (débits de tabac, académies de billard, barbiers). Le durcissement de la réglementation des débits de boissons en 1872 renforça cette tendance. Il semble toutefois que les autorités aient fait preuve d'une relative tolérance tant à l'égard des sweeps que des betting houses, dont la concurrence conduisit quelques bookmakers de Tattersall's à ouvrir des officines en ville. En outre, quand une campagne de presse entraînait des fermetures d'établissements, comme à Manchester et à Londres en 1869, certains bookmakers décidaient de publier leurs cotes dans la presse et de s'installer en Écosse, où la loi ne s'appliquait pas. Lorsque celle-ci fut révisée en 1874, on vit des « bureaux de paris au comptant » fleurir à l'étranger, notamment à Boulogne-surMer. Des clubs spécialisés se multiplièrent également, mais leur légalité était fragile s'il était prouvé qu'un professionnel des paris figurait parmi les membres ${ }^{47}$. La solution adoptée par un nombre croissant de bookies fut d'exercer leur activité en plein air. Dès 1859, les commerçants de Manchester se plaignaient de voir leurs rues encombrées par la 'betting nuisance' ${ }^{48}$. Le street betting ${ }^{49}$ se développa donc à partir du milieu du siècle dans les principales villes du pays. En 1876, selon certains journalistes, il s'était étendu à toutes les bourgades du pays ${ }^{50}$.

21 Cet engouement populaire était aussi le résultat d'un meilleur accès à l'information. Depuis les années 1850, le télégraphe électrique permettait de faire parvenir rapidement des informations aux rédactions. De nouveaux journaux à bon marché (Sporting Life, 1859; Sportsman, 1865 ; Sporting Chronicle, 1871) privilégiaient les courses hippiques et publiaient les «tuyaux » des pronostiqueurs (tipsters) afin d'augmenter leur tirage. La vogue des paris est donc indissociable du développement de la presse populaire. En 1893, 70 quotidiens, contre deux en 1870, offraient chaque soir des éditions spéciales contenant le résultat des courses sur lesquelles on avait parié le jour même. L'avancée la plus significative fut la publication dans la presse des cotes offertes au départ sur l'hippodrome (starting prices), plus fiables que celles des bookmakers urbains. Chaque bookmaker des rues convenait avec ses clients de s'aligner sur elles. Ce système présentait des garanties pour le parieur et renforçait la discrétion de la transaction. Il n'y avait plus lieu de discuter de la cote ou de l'afficher puisque celle-ci ne serait connue qu'après la course, dans les éditions du soir. Sa généralisation précipita le déclin du list betting au profit du street betting dans les années 1880 .

La procédure était très simple : dans un endroit convenu, désigné par le terme de pitch, le parieur remettait au bookmaker ou à son employé un morceau de papier, signé d'un pseudonyme, sur lequel il avait inscrit le nom du cheval choisi. Le pitch pouvait être un 
coin de rue, les abords d'une usine, un pub, une place isolée ou un terrain vague. La charge retenue pour réprimer ces agissements était celle d'obstruction de la voie publique. De nombreux arrêtés municipaux s'inspirèrent du Metropolitan Streets Act de 1867 (article 23), qui rendait passible d'amende tout attroupement dans la capitale d'au moins trois personnes en train de parier. Mais le montant de ces amendes (£5) n'était guère dissuasif. En regard du chiffre d'affaires des bookmakers, il ne s'agissait, même en cas de récidives multiples, que d'une formalité. Le renforcement en 1873 de la loi sur le vagabondage (Vagrant Act), qui servait initialement à réprimer le jeu de pile-ou-face ou certains jeux de cartes en plein air ${ }^{51}$, s'avéra tout aussi inefficace pour juguler un phénomène qui prenait de l'ampleur. On estime qu'en 1914, le nombre des parieurs réguliers était de quatre millions ${ }^{52}$, et ce chiffre ne tenait pas compte des occasions exceptionnelles comme le Derby ou le Grand National. Certains de ces paris étaient engagés sur l'hippodrome ou dans des officines légales, où l'on n'opérait qu'à crédit et par correspondance; mais dans leur immense majorité, il s'agissait de quelques pence ou shillings misés auprès d'un street bookie. Naguère confinés à une élite sociale et à quelques milieux interlopes ${ }^{53}$, les paris hippiques étaient devenus l'activité (masculine) de loisir la plus répandue dans les milieux ouvriers, mais en marge de la légalité.

Cette extension sans précédent suscita un regain d'inquiétude, voire de panique, autour du jeu dans les dix dernières années du siècle. A cela s'ajoutait sa plus grande visibilité. Les perquisitions nocturnes dans des lieux clos et discrets avaient cédé la place au spectacle de 'street-bookies' postés à l'entrée des usines à la pause de midi ${ }^{54}$, ou celui des vendeurs à la criée des éditions spéciales. De multiples articles, ainsi qu'un synode de l'Église anglicane, furent consacrés à un "fléau » où certains voyaient la marque d'un déclin national. Une Ligue nationale contre le jeu (NAGL), calquée sur le modèle des ligues de tempérance, fut fondée en 1890. Minimisant les pratiques plus anciennes, on s'accordait à dire que le jeu était un phénomène nouveau dans les classes populaires, où il constituait une menace, tant pour la famille que pour une société chrétienne et industrielle. Il semble au contraire que la majorité de ces nouveaux turfistes aient dépensé leur argent de façon raisonnable et calculée, à l'image des préceptes victoriens dominants ${ }^{55}$. En outre, la quête de bons «tuyaux » constituait un élément de sociabilité important qui rythmait la vie quotidienne, à l'atelier, à l'usine comme au pub ${ }^{56}$. La prohibition, renforcée en 1906 sous l'influence de la NAGL, fut vouée à l'échec car son caractère sélectif lui donna l'image d'une loi socialement injuste. Bénéficiant du soutien de la population, les bookmakers des rues et leurs guetteurs furent des personnages omniprésents dans les villes britanniques jusqu'aux réformes des années 1960. Avec leur succès et celui de la presse populaire, le monde des courses s'éloignait un peu plus de ses origines aristocratiques et rurales pour s'intégrer à une culture de masse résolument urbaine. 


\section{NOTES}

1. Voir notamment Goulven GUILCHER, « Avatars et agonie du prize fighting 1830-1860 », Cahiers victoriens et édouardiens, $\mathrm{N}^{\circ} 19$, avril 1984, pp. 23-31 ainsi que Dennis BRAILSFORD, Bareknuckles : A Social History of Prize-fighting, Cambridge: Lutterworth, 1988.

2. House of Commons Select Committee on Gaming, 1844, présidé par Palmerston ; House of Lords Select Committee on the Laws Respecting Gaming, 1844, présidé par le duc de Richmond.

3. On entend par paris urbains les paris engagés en dehors du champ de courses, quelle que soit l'importance de la localité.

4. House of Lords Select Committee on Betting, 1902, présidé par le comte de Durham.

5. Rappelons que l'appartenance à ces clubs, presque tous fondés au XVIII ${ }^{\mathrm{e}}$ siècle, reposait sur la cooptation et le versement de droits d'inscription et d'abonnement très élevés.

6. C'est la raison pour laquelle il utilise indifféremment les termes de 'public' ou de 'common' gambling house pour désigner les établissements de l'autre catégorie. Un responsable de la City Police Force confirme cette distinction en insistant également sur le caractère privé des clubs, circonstance atténuante à ses yeux. House of Commons, Minutes of Evidence Taken before the Select Committee on Gaming, 1844, questions 813-817 (D.W. Harvey, 7 March 1844).

7. Ibid., question 2856 (28 March 1844).

8. Ibid., questions 2847 et 2871 .

9. 'Every public gaming house that can be put down should be put down', ibid, question 2853.

10. Ibid, questions 62-65.

11. Ibid, questions 363 et 388 .

12. Ibid., question 970. La peine maximale encourue était de six mois d'emprisonnement. Pour un rapport détaillé, voir la déposition d'un des responsables de la police londonienne (Commissioner of the Metropolitan Police) le 9 mai 1844 (questions 3159-3245).

13. La déposition du commissaire $T$. Baker est extrêmement claire à ce sujet. Voir ibid., questions 482-487. Un de ses collègues en poste dans la City déclare (question 751) : 'There are many inferior public-houses and coffee-shops in the neighbourhood of Houndsditch, where very many Jews reside, in which gambling for very inconsiderable amounts is carried on, and their existence is generally brought under the notice of the police by anonymous letters, apparently from persons who may have lost their money.'

14. On faisait généralement valoir qu'il s'agissait de clubs «fictifs" ouverts à tous, l'élection étant de pure forme. D'autre part, les «véritables » clubs remplissaient plusieurs fonctions, ils n'étaient pas exclusivement des établissements de jeu.

15. Ibid., question 801. Rous tient un discours analogue : 'The poor should be protected, but I would let a rich man ruin himself if he pleases' (Ibid., question 2857). Voir également les questions 615-617.

16. Le droit d'inscription fut fixé à trente guinées, le plus élevé de Londres, et dès février 1828, un mois après l'ouverture, le Bell's Life in London pouvait déclarer que les adhérents du club comptaient parmi eux «la fine fleur de l'aristocratie" ('The most distinguished noblemen in the country are members of the club.'). Cité par Henry BLYTH, Hell and Hasard or William Crockford versus the Gentlemen of England, London: Weidenfeld \& Nicolson, 1969, p. 103.

17. Celui-ci déclare avoir jugé préférable de démissionner de Crockford's avant son mariage (House of Commons, op. cit., question 2864) avant d'exprimer son désir de voir le club détruit par un incendie (ibid., question 2868). D'autres témoignages allaient dans le même sens (ibid., questions 888-889). 
18. Cette porosité est confirmée par le compte rendu d'une perquisition dans un tripot clandestin (House of Lords, Minutes of Evidence Before Select Committee on the Laws Respecting Gaming, 1844, questions 63-70: 'Have you met any of the higher Class in any of theses Houses' - 'Yes; I am sorry to say I have'. - [...] 'Do you find that there are mixed Classes of Persons in the same Gaming House?' - 'Different Grades.'

19. Si dans les grands clubs, la désaffection était principalement due à la pression sociale, on peut supposer que la répression des autres établissements fut accrue. C'est ce que semblent indiquer les poursuites engagées contre les débits de boissons londoniens pour jeux prohibés dès le début de la décennie, avant la révision de la loi (21 en 1841, 31 en 1842, 36 en 1843). Les deux tiers de ces poursuites donnaient lieu à une condamnation (House of Commons, op.cit., appendix V).

20. Voir Mike HUGGINS, Flat Racing and British Society 1790-1914, London: Frank Cass, 2000, p. 57.

21. House of Lords, op.cit., questions 119-124.

22. House of Commons, op.cit., questions 1307-1396. Vers 1844, il y avait entre 10 et 12 baraques de jeux (gaming ou gambling booths) à Hampton, 16 ou 17 à Epsom. L'organisateur des courses de Goodwood n'en signalait que deux (questions 1158-1162), elles aussi tenues par des personnes venues de Londres, qui rapportaient 250 livres de loyer annuel, contre 50 pour les débits de boisson (refreshment booths) (questions 1227-1228).

23. " "The Aintree Carnival" \& "The Aintree Meeting" ', pp. 71-86 in John K. WALTON \& Alastair WILCOX (eds.), Low Life and Moral Improvement in Mid-Victorian England, Leicester: Leicester University Press, 1991.

24. House of Commons, op.cit. Plusieurs témoins confirment l'augmentation du nombre de spectateurs depuis quelques années (House of Lords, op. cit., questions 198-199; 266 et 317).

25. Ibid., questions 1020-1023.

26. HUGGINS, op. cit., p. 151.

27. Voir Carl CHINN, Better Betting with a Decent Fellow, Hemel Hempstead: Harvester Wheatsheaf, 1991, pp. 2-3 et 58 ; Emmanuel ROUDAUT, «Les Controverses sur le jeu dans la société britannique, 1890-1961 ", thèse de doctorat, Université de Lille 3, 1997 (collection "thèse à la carte ", Presses universitaires du Septentrion), pp. 12-13 ; Wray VAMPLEW, The Turf: A Social and Economic History of Horse-racing, London: Allen Lane, 1976, p. 70..

28. Voir par exemple House of Lords, op. cit., questions 240-241. Le scandale le plus retentissant fut celui du Derby de 1844. Voir BLYTH, op. cit., chapitres 7 et 8 ou Roger MORTIMER, The Jockey Club, London: Cassell, 1958, pp. 72-78.

29. Sporting Indicator and Turf Telegraphist, 14 June 1880, cité par David C. ITZKOWITZ, 'Victorian Bookmakers and their Customers', Victorian Studies, Vol.32, №1, Autumn 1988.

30. George MOORE, Esther Waters, Londres, 1894, chapitre 31.

31. House of Commons, op. cit., question 1020.

32. Op.cit., p. 36. Principales localités concernées : Bromley, Croydon, Enfield, Harrow, Kingsbury, Streatham et West Drayton.

33. James GREENWOOD, The Seven Curses of London, London: Stanley Rivers, 1869, pp. 399-401.

34. Ibid., pp. 402-403. De même, lorsque Rous oppose les gentlemen au 'swarm of locusts who will come in and bet any sum of money and never pay' c'est probablement aux bookmakers qu'il fait allusion (House of Commons, op. cit., question 2878). On peut penser que cette présentation contrastée est tout autant inspirée par des préjugés de classe que par des faits.

35. Ibid., questions 1042 et 1048. Le terme péjoratif de blackleg désignait les bookmakers au XIX ${ }^{\mathrm{e}}$ siècle.

36. HUGGINS, op.cit., pp. 182-185.

37. Voir par exemple House of Commons, op.cit., question 1252.

38. Voir Wray VAMPLEW, Pay Up and Play the Game, Cambridge: Cambridge University Press, 1988, p. 57. Sandown Park, bientôt imité par la plupart des autres champs de courses, se constitua en société anonyme dès 1885 . 
39. Cité par MORTIMER, op. cit. 116-117. La référence n'est pas précisée. Il y eut cependant un regain de violence au siècle suivant, pendant l'entre-deux-guerres. Voir Edward GREENO, War on the Underworld, London: Brown and Watson, 1959, ainsi que ROUDAUT, op. cit., pp. 225-229.

40. Kellow CHESNEY, The Victorian Underworld, London: Temple Smith, 1970, p. 290. Ce type de combat était encore très prisé sous le règne de Guillaume IV. Chesney évoque une rencontre importante organisée par le comte de Derby en 1830.

41. Henry MAYHEW, London Labour and the London Poor, vol. III, London: Woodfall, 1851, pp. 7 et suivantes, cité par CHESNEY, op. cit., pp. 294-300.

42. 'The Dog Fight' in WALTON \& WILCOX (eds.), op.cit.. pp. 65-70.

43. Ibid., p. 74. Pour une évocation du pédestrianisme victorien, voir le roman de Peter LOVESEY, Wobble to Death, London: Macmillan, 1970.

44. Voir HUGGINS, op. cit., p. 21.

45. Voir ITZKOWITZ, loc.cit., pp. 10-11; House of Commons, op. cit., questions 2492-2500; House of Lords, op. cit., questions 197-200, HUGGINS, op.cit., p. 99.

46. Hull Advertiser, 15 \& 22 August 1851, cité par CHINN, op. cit., p. 70.

47. On y retrouvait la distinction entre les établissements « respectables » et les autres : ' $a$ disreputable combination of gambling hell with the lowest type of dancing saloon', Charles BOOTH, Life and Labour of the People in London, $1^{\text {st }}$ series, London: Macmillan, 1902, p. 94, cité par CHINN, op. cit., p. 87.

48. Sporting Life, 21 September 1859.

49. Pour une étude approfondie, consulter CHINN, op. cit; Mark CLAPSON, A Bit of a Flutter : Popular Gambling in England c. 1820-1961, Manchester: Manchester University Press, 1991; David DIXON, From Prohibition to Regulation, Oxford: Clarendon Press, 1991 ; ainsi que l'article fondateur de Ross McKIBBIN, 'Working-class Gambling in Britain, 1880-1939', Past and Present, $\mathrm{N}^{\circ} 82$, February 1979.

50. 'The Ring and the Book', Baily's Magazine, March 1876, p.193.

51. Pour une description du pitch and toss, voir House of Commons, op. cit., questions 199-206, et CHINN, op.cit., pp. 63-64 et 101.

52. HUGGINS, op. cit., p. 105.

53. Notamment les fancy-men. Cf. Henry MAYHEW, London's Underworld (edited by Peter Quennell), London: Bracken Books, 1983, p. 106.

54. Select Committee of the House of Lords on Betting, 1901-1902, Minutes of Evidence, question 1849.

55. Voir McKIBBIN, loc.cit., ainsi qu'un article plus récent et plus ciblé : Mike HUGGINS, 'The first Generation of Street Bookmakers in Victorian England'. Northern History, Vol.36, N 1 , May 2000, pp. 129-145.

56. Voir par exemple Robert TRESSELL, The Ragged Trousered Philanthropists [1914] Londres: Paladin, 1991, pp. 17-18.

\section{RÉSUMÉS}

Le présent article est consacré aux inquiétudes liées au monde du jeu dans l'Angleterre victorienne. Dans les années 1840, une série de scandales donne lieu à une répression accrue des cercles de jeu, notamment dans la capitale. Un effort de moralisation des courses hippiques est 
également entrepris. A la fin du siècle, les hippodromes présentent une image assainie et assagie, mais l'attention se porte désormais sur de nouvelles formes de paris hippiques urbains. Rendue possible par l'essor de la presse et de nouvelles technologies, leur extension dans les milieux populaires suscite une nouvelle « panique morale».

This paper examines the concern expressed on the issue of gambling in Victorian England. In the 1840 s, a series of scandals led to an increase in the repression of gambling houses. Attempts were also made to moralise the turf. By the end of the century, the rowdiest and seediest aspects of race courses had been eliminated. However, attention now focused on new forms of off-course betting on horses, made possible by the rise of the popular press and the development of new technologies. The widespread success of 'street-betting' among the working class was the cause of a new 'moral panic' in late-Victorian years.

\section{AUTEUR}

\section{EMMANUEL ROUDAUT}

Université de Valenciennes 